\title{
Effects of prenatal alcohol exposure on hippocampal volume, verbal learning, and verbal and spatial recall in late childhood
}

\author{
KAREN A. WILLOUGHBY, ${ }^{1,2}$ ERIN D. SHEARD, ${ }^{1}$ KELLY NASH, ${ }^{1,3}$ AND JOANNE ROVET ${ }^{1,2,4}$ \\ ${ }^{1}$ Neuroscience and Mental Health Research Program, Hospital for Sick Children, Toronto, Ontario, Canada \\ ${ }^{2}$ Department of Psychology, University of Toronto, Toronto, Ontario, Canada \\ ${ }^{3}$ Department of Human Development and Applied Psychology, Ontario Institute for Studies in Education, \\ Toronto, Ontario, Canada \\ ${ }^{4}$ Department of Pediatrics, University of Toronto, Toronto, Ontario, Canada
}

(Received April 8, 2008; Final Revision July 26, 2008; AcCePted July 28, 2008)

\begin{abstract}
Children with prenatal alcohol exposure (PAE) show deficits in verbal learning and spatial memory, as well as abnormal hippocampal development. The relationship between their memory and neuroanatomic impairments, however, has not been directly explored. Given that the hippocampus is integral for the synthesis and retrieval of learned information and is particularly vulnerable to the teratogenic effects of alcohol, we assessed whether reduced learning and recall abilities in children with fetal alcohol spectrum disorders (FASDs) are associated with abnormal hippocampal volumes. Nineteen children with FASDs and 18 typically developing controls aged 9 to 15 years were assessed for verbal learning and verbal and spatial recall and underwent structural magnetic resonance imaging. Images were analyzed for total intracranial volume and for right and left hippocampal volumes. Results revealed smaller left hippocampi and poorer verbal learning and verbal and spatial recall performance in children with FASDs than controls, as well as positive correlations between selective memory indices and hippocampal volumes only in the FASD group. Additionally, hippocampal volumes increased significantly with age in controls only, suggesting that PAE may be associated with long-term abnormalities in hippocampal development that may contribute to impaired verbal learning and verbal and spatial recall. (JINS, 2008, 14, 1022-1033.)
\end{abstract}

Keywords: Hippocampus, Fetal alcohol spectrum disorders, Spatial recall, Verbal learning and recall, Magnetic resonance imaging, Adolescence

\section{INTRODUCTION}

Prenatal alcohol exposure (PAE) is one of the most common preventable causes of birth defects and cognitive impairment leading to a variety of clinical conditions described by the umbrella term, fetal alcohol spectrum disorders (FASDs; Kodituwakku, 2007; Niccols, 2007). FASDs are typically reported to affect between 0.3 and 4 children per thousand annually (Abel \& Sokol, 1991; May \& Gossage, 2001; Sampson et al., 1997; Stratton et al., 1996), with as many as 10 per 1000 in several native communities in the United States and Canada (Square, 1997) and one isolated Canadian community reporting FASDs in up to $20 \%$ of youth (Robinson

Correspondence and reprint requests to: Joanne Rovet, Department of Psychology, Rovet Lab, The Hospital for Sick Children, 555 University Avenue, Toronto, ON, Canada. E-mail: joanne.rovet@sickkids.ca et al., 1987). Among inner-city black South Africans, $6 \%$ to $7 \%$ of all offspring have an FASD (Viljoen et al., 2005) and in Italy, where moderate drinking is the norm, PAE affects $2 \%$ to $4 \%$ of all children (May et al., 2006). Because FASDs are commonly associated with life-long neurobehavioral consequences, the costs of FASDs are staggering at more than 1.4 million dollars per individual across his or her lifespan (Hardwood, 2000; Stade et al., 2007; Streissguth et al., 1991).

FASDs encompass fetal alcohol syndrome (FAS; Jones \& Smith, 1973) and alcohol-related neurodevelopmental disorder (ARND; Clarren \& Smith, 1978; Riley \& McGee, 2005), as well as several other associated conditions (e.g., fetal alcohol effects, alcohol-related birth defects, and partial FAS). FAS is characterized by a triad of features, including a distinctive facial dysmorphology, growth retardation, and severe neurobehavioral problems (Jones \& Smith, 1973). In contrast, ARND refers to neurobehavioral and cognitive 
manifestations in the absence of typical FAS dysmorphic features and is observed in most children with confirmed PAE (Mattson et al., 1998; Stratton et al., 1996). Evidence from animal research suggests that the extent and severity of deficits associated with FASDs may depend on several factors, such as amount, timing, and duration of PAE, and variation in these factors can contribute to distinctly different outcomes (Berman \& Hannigan, 2000; Goodlett \& Johnson, 1999; Tomlinson et al., 1998).

Because of the variability in deficits associated with PAE, no single neuropsychological profile of FASDs accurately describes every affected individual. Rather, individuals with a FASD often demonstrate a wide range of long-term cognitive and behavioral impairments, which include learning and memory deficits, attention and executive function problems, poor visuospatial skills, and reduced IQ (for a review, see Kodituwakku, 2007; Mattson \& Riley, 1998; Niccols, 2007). As a result of this variability, much research to date has focused on delineating the behavioral and cognitive phenotype of FASDs, as well as characterizing the underlying neuroanatomy associated with this phenotype in order to provide critical information for improving early diagnosis of FASDs and constructing more effective interventions.

In FASDs, one of the more commonly reported neuropsychological deficits is impaired learning and memory, particularly deficits in verbal learning and verbal and spatial recall (Mattson et al., 1996; Uecker \& Nadel, 1996). For example, Willford et al. (2004) showed that children with moderate PAE had impairments in both immediate and delayed recall on the Word Pairs subtest of the Children's Memory Scale (CMS). The effects of PAE on their verbal recall, however, were mediated by their verbal learning performance, such that children with poorer acquisition of new information had the larger deficits in verbal recall (Willford et al., 2004). In other studies using the California Verbal Learning Test-Children's Version (CVLT-C), Mattson and colleagues observed that pervasive verbal learning deficits in children with PAE contributed to their impaired delayed verbal recall ability (Mattson et al., 1996, 1998; Mattson \& Roebuck, 2002). Taken together, these findings suggest children with FASDs have difficulty acquiring new information, and this consequently appears to have an impact on other aspects of memory function.

Current understanding of spatial memory deficits in children with FASDs is largely derived from an extensive animal literature showing that rats exposed to ethanol during early brain development had impaired place learning ability (Gianoulakis, 1990; Sutherland et al., 2000) and damage to the hippocampus (Klintsova et al., 2007; Livy et al., 2003), an area closely associated with spatial learning and recall and particularly vulnerable to alcohol exposure in animals and humans (Berman \& Hannigan, 2000). In a study of three children who sustained early bilateral hippocampal damage due to hypoxic-ischemic brain injury, VarghaKhadem et al. (1997) found significant impairments in delayed reproduction of a spatial arrangement using the Rey-Osterrieth Complex Figure task (ROCF) and poor everyday spatial memory using the parental report Everyday Memory Questionnaire (EMQ). Although relatively few studies have directly examined spatial recall ability in children with PAE, a report by Uecker and Nadel (1996) indicated impaired recall for spatial locations in children with FAS, who had difficulty reproducing previously viewed spatial arrangements both immediately and after a delay and also made more distorted reproductions than controls (Uecker \& Nadel, 1996). Furthermore, Uecker \& Nadel (1996) suggested that the spatial deficits observed in children with FAS may be particularly associated with right hippocampal damage because their spatial deficits were similar to those exhibited by patients with right temporal lobectomies and extensive right hippocampal lesions. Consistent with their hypothesis, Bohbot et al. (1998) found that patients with right, but not left, hippocampal damage were impaired on the immediate and delayed conditions of the ReyOsterrieth Figure Task (ROCF), suggesting that the right hippocampus may play an important role in visuospatial learning and recall. Overall, these findings suggest that although PAE has widespread neurobehavioral consequences, specific disruptions in hippocampal development (particularly in regard to the right hippocampus) may be associated with difficulties in spatial learning and recall.

Recent use of functional magnetic resonance imaging (fMRI) techniques provides the opportunity to investigate whether verbal learning and verbal and spatial recall deficits in children with FASDs are particularly associated with alcohol-related damage to the hippocampus and other medial temporal lobe structures. Using an fMRI verbal learning paradigm, Sowell et al. (2007) found that in contrast to controls who exhibited unilateral activation in left medial temporal structures (i.e., the hippocampus and parahippocampal gyri), children with PAE showed a complete absence of activation in the same medial temporal region, even after controlling for group differences in memory performance. This result suggests that children with PAE may have a dysfunctional medial temporal memory network, which requires compensation from additional brain areas (Sowell et al., 2007). Although functional neuroimaging studies using positron emission tomography (PET) have demonstrated clear hippocampal activations during virtual tests of spatial navigation in typically developing children (Ghaem et al., 1997; Maguire et al., 1998), this has yet to be investigated in children with FASDs. In fact, apart from the study by Sowell et al. (2007) and one examining spatial working memory in children with FASDs (Malisza et al., 2005), no study to our knowledge has yet examined the relation between learning and memory impairments and abnormalities in brain activation in children with FASDs.

Studies using structural neuroimaging techniques have shown that children with FASDs have abnormalities in the size and composition of the hippocampus and temporal lobe. Archibald et al. (2001) reported mild reductions in hippocampal volume that were slightly larger in children with FAS than children with PAE and no facial dysmorphology. However, these reductions in hippocampal volume were 
proportional to reductions in overall brain size (Archibald et al., 2001). Additional differences in hippocampal architecture between children with PAE and controls were found by Riikonen et al. (1999, 2005), who reported reduced hippocampal volumes and greater hippocampal asymmetry (i.e., left smaller than right) in children with PAE relative to controls, as well as by Autti-Rämö et al. (2002), who found left hippocampal thinning in three children with varying levels of PAE. Several studies of PAE in children have used structural MRI to investigate the structure-function correlations between verbal learning and recall and the corpus callosum (Sowell et al., 2001), cerebellum (O'Hare et al., 2005), as well as overall cortical thickness (Sowell et al., 2008). No study, however, has directly examined whether the deficits in verbal learning and verbal and spatial recall in this clinical population are directly related to abnormalities in hippocampal development. Thus, large gaps still remain in our understanding of the cognitive and associated neuroanatomic manifestations of FASDs, particularly with regard to verbal and spatial learning and recall.

The present study therefore sought to extend our knowledge of FASDs by investigating verbal learning and verbal and spatial recall in children with FASDs from behavioral and neuroanatomic perspectives, using a clinical neuropsychological battery of learning and recall tests, as well as structural MRI. This study had three main aims: (i) to compare children with FASDs and typically developing controls on tests of verbal and spatial learning and recall, (ii) to compare these groups on hippocampal volume and changes in hippocampal volume across age, and (iii) to determine whether children with FASDs showed a distinctive pattern of structure-function correlations relative to controls. We hypothesized that the FASD group would show impaired verbal learning, as well as poor verbal and spatial recall. Given earlier findings of left-right hippocampal asymmetries (i.e., smaller left than right volume, Riikonen et al., 1999, 2005) and left hippocampal thinning (Autti-Rämö et al., 2002) in children with PAE, we hypothesized that any reductions found in hippocampal volume may be greater in the left than the right hippocampus in children with FASDs. We further hypothesized that spatial recall deficits found in children with FASDs would be more strongly associated with right than left hippocampal volume reductions, based on studies relating poor spatial recall to right hippocampal damage (Bohbot et al., 1998; Uecker \& Nadel, 1996). Finally, we expected to find structure-function correlations such that any abnormalities in hippocampal volume found within the FASD group would be correlated with impairments in verbal and spatial learning and recall.

\section{METHODS}

\section{Research Participants}

Participants included 19 children (14 boys $/ 5$ girls) aged 9.3 to 15.4 years with a FASD originally diagnosed by the clin- ical team in the Motherisk Follow-up Clinic at the Hospital for Sick Children (SickKids). Motherisk is a diagnostic service for FAS or ARND based on the Canadian guidelines and standards (Chudley et al., 2005). To receive a diagnosis on the fetal alcohol spectrum, children must have had a documented history of PAE substantiated by maternal verification, alcohol related diagnosis/treatment during pregnancy, and/or removal by the Children's Aid Society (CAS) because of the biological mother's alcohol abuse or her heavy drinking during pregnancy. In most cases, documentation of PAE was obtained through CAS foster or adoption records or in statements made by the biological mother or a relative. Most children attending the clinic were brought in by foster parents, adoptive parents, CAS case workers, or in a minority of cases, a biological parent or relative. The diagnosis was made by qualified staff consisting of a board certified pediatrician, specifically trained and experienced in FAS diagnosis who performed the neurological and physical assessments and evaluated facial features for dysmorphology (scored on a three-point scale ranging from 1-absent to 3-highly present), and a registered psychologist and a psychometrist, who conducted the neuropsychological evaluation (for more details about the Motherisk diagnostic process see Greenbaum et al., 2002). In the current sample, only three children had facial dsymorphology consistent with a diagnosis of FAS and all others qualified for a diagnosis of ARND.

Control participants were 18 typically developing children aged 9.7 to 14.8 years ( 7 boys $/ 11$ girls) recruited from a pool of previously contacted control participants in the lab or in one case, the two biological children of a participating adoptive parent. No mother of any control children reported drinking alcohol during her pregnancy. Exclusionary criteria were: confirmed primary exposure to a teratogen other than alcohol in the FASD group or to any teratogenic substance in controls, a head injury or other neurological or brain abnormalities, a debilitating or chronic medical condition, and MRI contraindications such as wearing braces or other metal devices. We also excluded from our control group any child with a psychiatric diagnosis, including attention-related disorders, or an identified learning disability. Parents or caregivers provided written consent for participation in this study, and all participants provided verbal assent. All procedures were approved by the Research Ethics Board of The Hospital for Sick Children.

\section{Tests and Measures}

Parents/caregivers of all children participating in the study completed a structured case history form, which yielded demographic information about the child's prenatal, birth, developmental, and familial history. All children were assessed for intelligence using the Vocabulary and Matrix Reasoning subtests from the Wechsler Abbreviated Scale of Intelligence (WASI; Wechsler, 1999), which yields an estimate of Full Scale IQ. Verbal learning and recall were assessed using selective subtests from the Children's Mem- 
ory Scale (CMS; Cohen, 1997), a comprehensive measure of children's memory encoding, storage, and retrieval abilities, and the California Verbal Learning Test-Children's Version (CLVT-C; Delis et al., 1994), a test of verbal listlearning ability. Spatial memory was assessed using the ReyOsterrieth Complex Figure task (ROCF; Osterrieth \& Rey, 1944; Rey, 1942) and the Spatial Memory subscale of the Everyday Memory Questionnaire (EMQ; Isaacs et al., 2000; Sunderland et al., 1983; Vargha-Khadem et al., 1997). For each measure, raw performance scores were used.

For the CMS, all participants received the Stories and Word Pairs subtests. CMS Stories required participants to listen to two stories and after each, recall the story verbatim immediately and after a 30-minute delay, following which they had to recognize accurate story information from foils in a series of yes/no questions. CMS Word Pairs required participants to learn a list of 14 unrelated pairs of words over three trials and then recall as many pairs of words as possible immediately and after a 30-minute delay, following which they had to recognize true from false word pairs. Indices of immediate recall, delayed recall, and delayed recognition were derived from the raw performance scores for both subtests; however, because the scoring procedure differed depending on participants' age, raw performance scores from CMS Stories immediate and delayed recall tasks were first age-corrected. In addition, a measure of verbal learning derived from the initial three learning trials of the Word Pairs subtest was included in the analysis.

For the CLVT-C, participants were required to learn a list of 15 "shopping" items (List A) and recall as many items as possible after each of five presentations of the list. A distracter list (List B) was then presented after the fifth presentation of List A, following which participants had to recall words from List $\mathrm{A}$ in a free and then a cued recall manner (short-delay trials). After a 20-min delay, participants again recalled List $\mathrm{A}$ in free and cued recall manners (long-delay trials) and then had to complete a yes/no recognition test. Based on previous research investigating CVLT-C performance in pediatric clinical populations using Donders' (1999) 5-factor model (Griffiths et al., 2006; Salorio et al., 2005), and Mattson and Roebuck's (2002) analysis of CVLT-C performance in children with PAE, the following CVLT-C variables were analyzed from raw performance scores: trial 1 of List A ("attention span"), trial 5 of List A ("learning efficiency"), total trials of List A ("total learning"), short-delay free and cued recall, long-delay free and cued recall, and delayed recognition.

The ROCF required participants first to copy a complex figure shown on a stimulus card and then to recreate it after a 30-minute delay with the stimulus card no longer present. For each condition, drawing accuracy was scored using a comprehensive 36-point scale (Spreen \& Strauss, 1998). Only the delayed recall condition was analyzed in the present study. The EMQ is a 28 -item parental-report questionnaire, which assesses long-term memory in everyday life situations on a 9-point scale ranging from 1 (not at all in the last 3 months) to 9 (more than once a day). Using the factor structure outlined by Cornish (2000), a composite measure of spatial recall ability was created using four items assessing everyday spatial memory (e.g., "getting lost where he/she has often been before").

\section{Procedure}

Participants were seen on a single day in the SickKids Psychology Department. They were first assessed for cognitive functioning and immediately following, underwent structural MRI scanning in a 1.5 Tesla GE scanner in the Diagnostic Imaging Unit. During the 1-hour scanning session, children viewed movies via MRI-compatible goggles. Images used for volumetric analysis were first acquired using a gradient-echo (SPGR) T1-weighted series with $\mathrm{TR}=24 \mathrm{~ms}$, $\mathrm{TE}=5 \mathrm{~ms}$, flip angle $=45^{\circ}$, field of view of $24 \mathrm{~cm}$, slice thickness of $1 \mathrm{~mm}$, no gaps. The second and third series were a fast spin-echo (FSE) acquisition yielding two separate image sets: $\mathrm{TR}=3000 \mathrm{~ms}$, $\mathrm{TE}=17 \mathrm{~ms}$ and $\mathrm{TR}=$ $3800 \mathrm{~ms}, \mathrm{TE}=102 \mathrm{~ms}$, slice thickness $=4 \mathrm{~mm}$, no gaps. On completion of the session, children received a movie pass, certificate of participation, and $\mathrm{CD}$ and printed picture of their brain. A neuroradiologist masked to group status examined all MRI images qualitatively for any evidence of neuroradiological abnormalities.

MRI image post-processing involved extraction of the brain from the skull and CSF and normalizing to standard space using anterior and posterior commissure alignment. Two independent raters masked to group status manually traced the left and right hippocampi (see Fig. 1) while one rater additionally traced the entire intracranial area (i.e., including all gray and white matter and cerebrospinal fluid) using ANALYZE 6.0 software. Designation of the hippocampal region included the volumes of the dentate gyrus, hippocampus proper, and subicular complex. Hippocampal boundaries were outlined slice by slice by a trackballdriven cursor from anterior to posterior in coronal images, such that designation began at the rostral end when the hippocampal head first appeared below the amygdala and ended when the crura of the fornices depart from the hippocampal tail at the caudal end. Further boundary verification was conducted from sagittal images in order to delineate the inferior boundary of the hippocampus from white matter from the parahippocampal gyrus. Interrater reliability was $98.8 \%$ for left hippocampal volumes and $99.9 \%$ for right hippocampal volumes.

\section{RESULTS}

\section{Demographic and Cognitive Function Measures}

Table 1 presents demographic information, including mean IQ scores for the FASD group and controls. A significant group difference was found for gender, $\left[\chi^{2}(1)=4.56, p<\right.$ $.05]$, reflecting more boys than girls in the FASD group. 


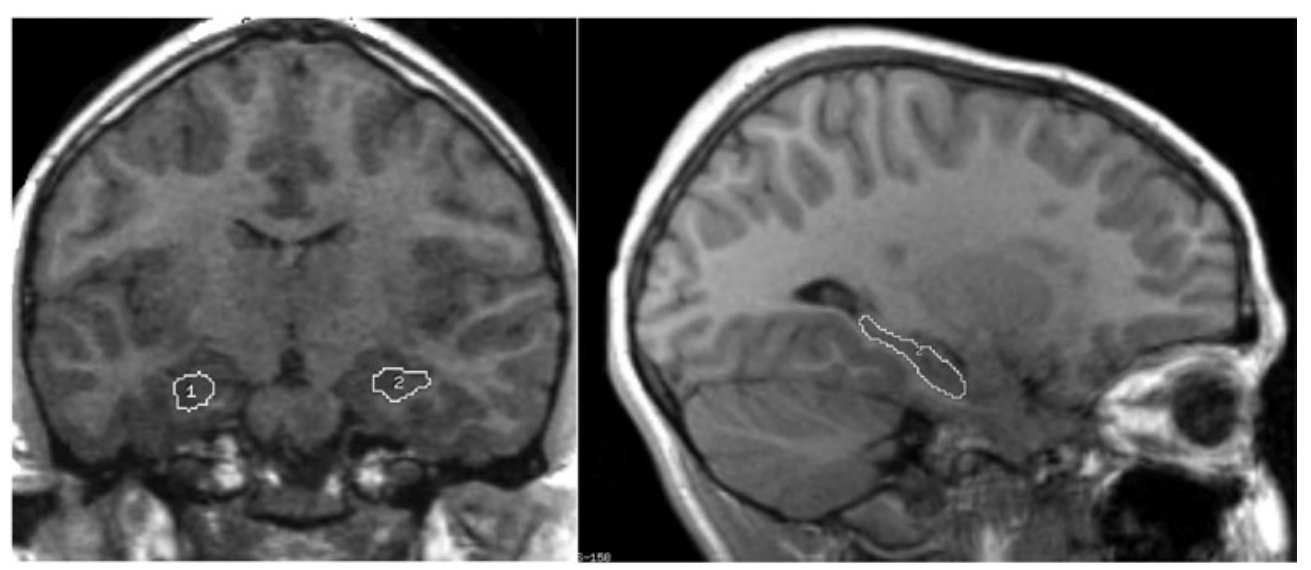

Fig. 1. Coronal (at slice 122) and sagittal (at slice 158) views of hippocampal tracings using ANALYZE 6.0 software. The left hippocampus is labeled as object 1 and the right hippocampus is labeled as object 2 (neurological view).

After controlling for age and gender using ANCOVA, FASD participants demonstrated significantly lower IQ scores than controls $\left[F(1,33)=50.44, p<.001 ; \eta^{2}=0.86\right]$. Groups did not differ in hand preference. At the time of the study, all controls were living with their biological parents, whereas the majority of FASD participants were living in foster or adoptive care. Several environmental factors significantly differed between the groups, including total family income, maternal caregiver age, and maternal caregiver education level (trend only). In addition, FASD participants had sig-

Table 1. Demographic information for FASD and control groups

\begin{tabular}{|c|c|c|c|}
\hline & & Group & \\
\hline & FASD & Controls & $p$ value \\
\hline Total number of participants & 19 & 18 & \\
\hline Mean age (years) & 12.21 & 12.39 & $n s$ \\
\hline Gender $(1=$ male, $0=$ females $)$ & 0.74 & 0.39 & $p<.05$ \\
\hline Mean Full Scale IQ and standard deviation (SD) & $83.11(17.29)$ & $119.89(11.07)$ & $p<.001$ \\
\hline IQ range & $56-113$ & $99-137$ & \\
\hline Handedness & & & \\
\hline Right & 13 & 12 & \\
\hline Left & 3 & 2 & \\
\hline Unable to determine (no response) & 3 & 4 & \\
\hline Participants in & & & \\
\hline maternal biological care & 1 & 18 & \\
\hline biological relative care (grandmother) & 1 & 0 & \\
\hline foster care & 12 & 0 & \\
\hline adoptive care & 5 & 0 & \\
\hline Total family income [Range: $1(\$ 30,0000)-5(\$ 120,000+)]$ & $2.73(1.10)$ & $4.00(0.85)$ & $p<.01$ \\
\hline Mean maternal caregiver age & $49.67(8.22)$ & $42.88(4.40)$ & $p<.01$ \\
\hline Mean maternal caregiver education level (Range: $1-5$ ) & $3.00(0.93)$ & $3.59(0.87)$ & $p<.08$ \\
\hline Mean birth weight (SD) & $3087.70(350.35)$ & $3585.14(557.21)$ & $p<.05$ \\
\hline Participants with & & & \\
\hline confirmed history of physical abuse & 6 & 0 & \\
\hline confirmed history of neglect & 12 & 0 & \\
\hline secondary prenatal exposure to cigarettes & 4 & 0 & \\
\hline secondary prenatal exposure to cocaine & 4 & 0 & \\
\hline a diagnosis of ADHD & 11 & 0 & \\
\hline Current Medications & & & \\
\hline Ritalin for ADHD & 3 & 0 & \\
\hline Concerta for ADHD & 4 & 0 & \\
\hline Clonidine for ADHD & 1 & 0 & \\
\hline Strattera for ADHD & 1 & 0 & \\
\hline Nexium \& 6-MP for Crohn's Disease & 0 & 1 & \\
\hline
\end{tabular}


nificantly smaller birth weights and a higher incidence of early physical abuse and neglect than controls.

Because both age and gender factors are known to affect brain size and some cognitive functions, all data were analyzed with age and gender as covariates in order to minimize their influence on between-group comparisons. In view of the exploratory nature of the study, an alpha of .05 was used without correction for multiple group comparisons of memory function. However, effect sizes are reported. Furthermore, multivariate analysis of covariance substantiated significantly lower scores in the FASD group compared to the control group across all CMS $[\mathrm{F}=5.25, p<.001]$ and all CVLT-C $[\mathrm{F}=3.91, p<.01]$ scores. Although the study focuses on results for individual measures, group differences were thus evident when controlling for alpha inflation.

For CMS Stories and Word Pairs, significant group differences were found on measures of immediate and delayed verbal recall, delayed recognition, and verbal learning, with controls significantly outperforming the FASD group on every measure (see Table 2). To examine whether learning ability accounts for significant group differences in verbal recall, a separate ANCOVA was conducted on immediate and delayed Word Pairs recall after covarying the number of word pairs initially learned on the three learning trials. Results revealed significant group differences in immediate $\left[F(1,32)=9.41, p<.01 ; \eta^{2}=0.12\right]$ and delayed $[F(1,32)=$ $\left.7.83, p<.01 ; \eta^{2}=0.13\right]$ recall conditions, indicating that FASD participants recalled fewer learned word-pairs than controls immediately and after a delay, even after controlling for initial learning.

On the CVLT-C, significant group differences were found in attention span, learning efficiency, total learning, and long-delay cued recall, whereas trends were found for shortdelay free recall, long-delay free recall and delayed recognition, with controls outperforming the FASD group on every measure (see Table 2). Similar to Mattson and Roebuck (2002), separate ANCOVAs were conducted on CVLT-C long-delay free and cued recall scores after first controlling for learning efficiency. These results indicated that learning ability accounts for the significant and trend-level group differences in CVLT-C long-delay recall scores since FASD participants remembered as much information as controls after correcting for initial learning $(p>.05)$. Finally, controls performed significantly better than the FASD group on the ROCF delayed spatial recall task and exhibited better everyday spatial recall on the EMQ.

\section{Brain Volume Measures}

Mean intracranial and hippocampal volumes of FASD and control groups are shown in Table 3. Using ANCOVA with age and gender controlled, we found a significant group difference in intracranial volume $[F(1,33)=8.14, p<.01$; $\left.\eta^{2}=0.16\right]$ due to the smaller volumes for the FASD group relative to controls. Furthermore, a significant effect of gender on intracranial volume was found for both FASD participants $\left[F(1,16)=4.70, p<.05 ; \eta^{2}=0.22\right]$ and controls $\left[F(1,15)=6.48, p<.05 ; \eta^{2}=0.30\right]$, with boys having significantly larger intracranial volumes than girls in both groups (see Table 3).

Given that FASDs have often been associated with significantly smaller intracranial volumes (Archibald et al., 2001; Sowell et al., 2002a), most of neuroimaging studies (including the current one) have examined proportional hip-

Table 2. Neuropsychological functioning by group and group comparisons using raw scores

\begin{tabular}{|c|c|c|c|c|}
\hline Test & $\begin{array}{c}\text { FASD } \\
M(S D)\end{array}$ & $\begin{array}{l}\text { Controls } \\
M(S D)\end{array}$ & F Statistic & $\begin{array}{l}\text { Effect Size } \\
\quad\left(\eta^{2}\right)\end{array}$ \\
\hline CMS stories immediate recall & $34.73(20.90)$ & $60.22(15.17)$ & $13.86^{* * *}$ & 0.27 \\
\hline CMS stories delayed recall & $28.90(16.23)$ & $46.01(20.33)$ & $17.94 * * *$ & 0.33 \\
\hline CMS stories delayed recognition & $22.42(3.92)$ & $26.61(1.65)$ & $12.97 * * *$ & 0.26 \\
\hline CMS word pairs immediate recall & $5.27(2.73)$ & $8.67(1.61)$ & $16.83 * * *$ & 0.33 \\
\hline CMS word pairs delayed recall & $3.90(2.60)$ & $7.56(2.43)$ & $15.40 * * *$ & 0.32 \\
\hline CMS word pairs delayed recognition & $38.59(4.50)$ & $41.44(0.92)$ & $4.83^{*}$ & 0.13 \\
\hline CMS word pairs learning & $20.34(7.10)$ & $27.94(7.60)$ & $5.89 *$ & 0.14 \\
\hline CVLT-C attention span (Trial1, List A) & $5.17(2.26)$ & $8.78(1.77)$ & $20.15 * * *$ & 0.40 \\
\hline CVLT-C learning efficiency (Trial 5, List) & $10.22(4.21)$ & $13.67(0.91)$ & $5.98 *$ & 0.16 \\
\hline CVLT-C total learning (Total Trials, List A) & $41.83(14.90)$ & $60.00(4.97)$ & $17.69 * * *$ & 0.35 \\
\hline CVLT-C short-delay free recall & $9.44(4.15)$ & $12.61(1.98)$ & $3.81^{\mathrm{t}}$ & 0.11 \\
\hline CVLT-C short-delay cued recall & $10.00(4.00)$ & $12.56(2.01)$ & 2.71 & 0.07 \\
\hline CVLT-C long-delay free recall & $9.53(4.05)$ & $12.28(1.87)$ & $3.60^{t}$ & 0.09 \\
\hline CVLT-C long-delay cued recall & $9.99(3.59)$ & $12.83(1.65)$ & $5.02 *$ & 0.10 \\
\hline CVLT-C delayed recognition & $13.23(2.55)$ & $14.72(0.58)$ & $3.45^{\mathrm{t}}$ & 0.09 \\
\hline ROCF delayed spatial recall & $9.92(9.31)$ & $23.19(7.40)$ & $16.21 * * *$ & 0.31 \\
\hline EMQ spatial memory & $13.81(8.49)$ & $5.61(2.06)$ & $11.94 * *$ & 0.28 \\
\hline
\end{tabular}

Note. Higher scores on the EMQ indicate poorer memory ability; $* * *=p<.001, * *=p<.01, *=p<.05,{ }^{\mathrm{t}}=p<.08$; two-tailed; CMS $=$ Children's Memory Scale, CVLT-C $=$ California Verbal Learning Test-Children's Version, ROCF $=$ Rey-Osterrieth Complex Figure task, EMQ = Everyday Memory Questionnaire). 
Table 3. Intracranial and hippocampal volumes $\left(\mathrm{mm}^{3}\right)$ by group and gender

\begin{tabular}{llcr}
\hline \hline & & \multicolumn{3}{c}{ Volumes $\left(\mathrm{mm}^{3}\right)$} \\
\cline { 2 - 4 } & \multicolumn{1}{c}{ Intracranial area } & Left hippocampus & Right hippocampus \\
Group & $M(S D)$ & $M(S D)$ & $M(S D)$ \\
\hline FASD (all participants) & $1416103.07(140081.90)$ & $1889.70(227.86)$ & $1882.68(278.20)$ \\
Boys $(N=14)$ & $1454838.14(127139.80)$ & $1875.77(253.84)$ & $1876.27(298.70)$ \\
Girls $(N=5)$ & $1307645.20(125750.40)$ & $1928.71(147.12)$ & $1900.64(240.34)$ \\
Controls $($ all participants $)$ & $1482683.06(113729.60)$ & $2036.39(249.06)$ & $1952.21(268.20)$ \\
Boys $(N=7)$ & $1558724.71(70500.96)$ & $2055.90(193.83)$ & $1984.36(171.21)$ \\
Girls $(N=11)$ & $1434292.91(111278.40)$ & $2023.98(287.18)$ & $1931.77(321.74)$ \\
\hline
\end{tabular}

pocampal volumes after first correcting for total intracranial volume (for a review of intracranial normalization methods in typically developing individuals, see Van Petten, 2004). Because of our specific directional hypothesis regarding the effects of PAE on hippocampal volume, subsequent data are presented using one-tailed tests for significance after controlling for age, gender, and intracranial volume. Results from a repeated measures ANCOVA indicated a significant group by hippocampal side interaction $[F(1,32)=$ $3.78, p<.05]$. Post-hoc $t$-tests revealed that FASD participants had significantly smaller left hippocampal volumes than controls $\left[t(35)=1.87, p<.05 ; \eta^{2}=0.09\right]$; (see Fig. 2), but did not differ in right hippocampal volume. This effect remained significant even after removing a FASD participant with a left hippocampal volume almost 2 standard deviations below the group mean.

In order to investigate the association between age and hippocampal volume in our cross-sectional sample, we corrected for total brain size by computing hippocampal volume as a proportion of total intracranial volume. In controls, age was positively correlated with left $(r=.62, p<.01)$ and right $(r=.46, p<.05)$ hippocampal volumes, whereas in the FASD group, an increase in hippocampal volume with age was not observed (see Fig. 3 and Table 4). Intracranial volumes did not change with age in either FASD or control groups.

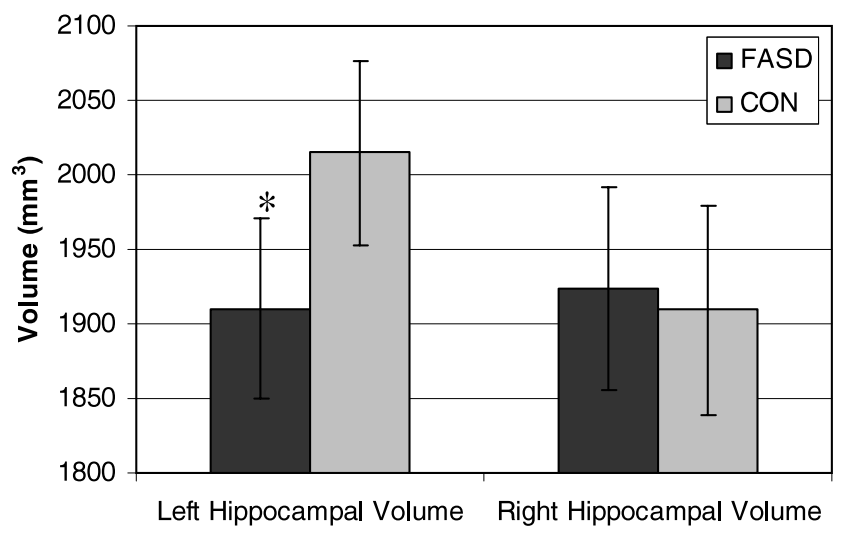

Fig. 2. Group differences in left and right hippocampal volume after controlling for age, gender, and total intracranial volume (*=significant group difference at $p<.05$; one-tailed).
While the goal of the present study was to collect a sample of children who were exposed only to alcohol, this was not feasible. Within our FASD group, four participants had additional prenatal exposure to cigarettes and a separate four participants had suspected secondary exposure to cocaine. Subgroup analyses indicated no significant difference between children with PAE only versus those with additional exposure to either cigarettes or cocaine on measures of hippocampal volume, birth weight, IQ, verbal learning, or verbal and spatial recall. For intracranial volume, however, a trend was found, $[F(1,13)=3.58, p<.10]$, reflecting the slightly smaller intracranial volumes of children with PAE and cocaine exposure than those with PAE only. Many FASD participants also had a diagnosis of attention deficit hyperactivity disorder (ADHD) and were taking medications for this condition; however, comparison of FASD participants with ADHD versus without ADHD revealed no significant difference on any of the dependent measures.

\section{Structure-Function Relationships}

Given that FASD participants have significantly reduced IQ scores and that age correlated significantly with hippocampal volume only in controls, it is possible that any significant correlations found between hippocampal volume and memory performance could be mediated by other underlying factors, such as IQ or age. Due to this possibility, we first determined the zero-order correlations between IQ, age, and all measures of learning and recall for both FASD and control groups (see Table 4). As shown in Table 4, IQ was highly correlated with the majority of measures of learning and recall in FASD participants, and age was highly correlated with ROCF delayed spatial recall in controls. Given these results, we then examined within-group partial correlations between hippocampal volume and memory function after controlling for IQ and age in order to identify which aspects of learning and recall were associated with left and right hippocampal volumes and determine whether the correlations differed between groups (see Table 4). Hippocampal volumes were analyzed as proportions of total intracranial volume to control for variability in intracranial size within each group. 


\section{A. Left Hippocampal Volume by Age in Controls}

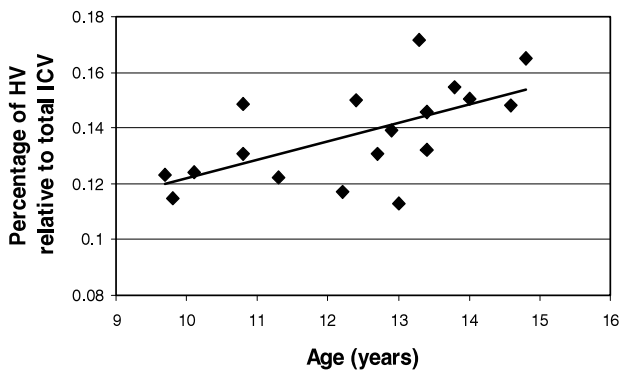

C. Left Hippocampal Volume by Age in FASD

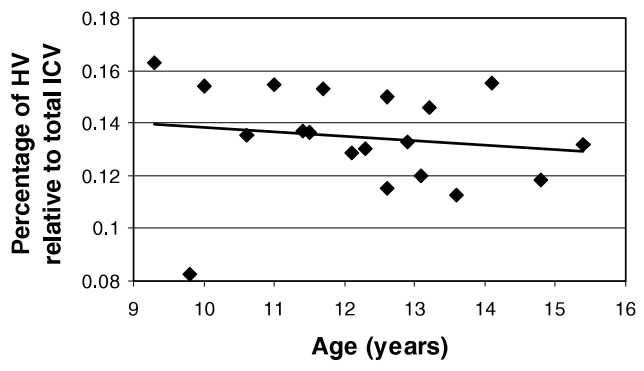

B. Right Hippocampal Volume by Age in Controls

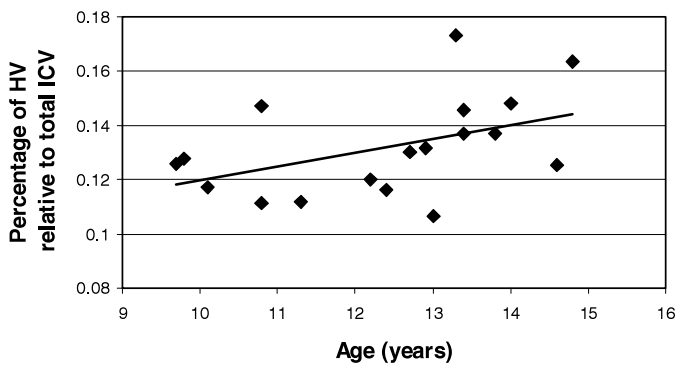

D. Right Hippocampal Volume by Age in FASD

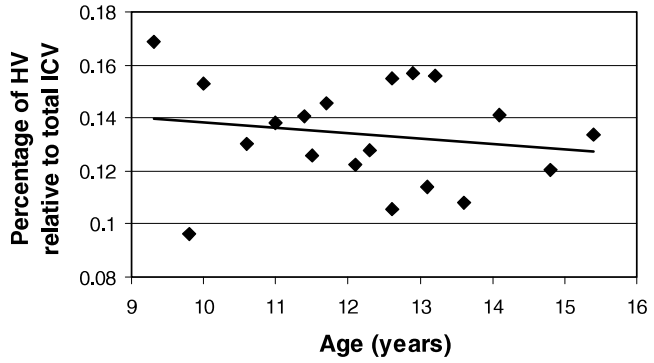

Fig. 3. Left and right hippocampal volume (presented as proportion of total intracranial volume) by age scatterplots for Controls (top graphs A and B) and FASD (bottom graphs C and D).

Table 4. Within-group zero-order correlations between IQ, age, hippocampal volume, and memory function (i.e., first four columns), and partial correlations between left and right hippocampal volumes and memory function after controlling for age and IQ (i.e., last four columns)

\begin{tabular}{|c|c|c|c|c|c|c|c|c|}
\hline \multirow[b]{2}{*}{ Test } & \multicolumn{2}{|c|}{ IQ } & \multicolumn{2}{|c|}{ Age } & \multicolumn{2}{|c|}{ Left HV } & \multicolumn{2}{|c|}{ Right HV } \\
\hline & FASD & $\mathrm{CON}$ & FASD & $\mathrm{CON}$ & FASD & $\mathrm{CON}$ & FASD & $\mathrm{CON}$ \\
\hline Left Hippocampal Volume (HV) & .244 & -.152 & -.149 & $.619 * *$ & - & - & - & - \\
\hline Right Hippocampal Volume (HV) & .163 & -.211 & -.168 & $.458 *$ & - & - & - & - \\
\hline CMS Stories Immediate Recall & $.631 * *$ & $.461 *$ & -.212 & -.363 & .132 & .046 & -.043 & .112 \\
\hline CMS Stories Delayed Recall & $.745 * * *$ & $.397^{t}$ & -.196 & -.372 & .406 & .279 & .246 & .342 \\
\hline CMS Stories Delayed Recognition & $.682 * * *$ & .117 & -.275 & -.198 & -.242 & .136 & -.485 & .096 \\
\hline CMS Word Pairs Immediate Recall & $.410^{t}$ & .236 & .245 & .030 & -.124 & -.201 & -.144 & .062 \\
\hline CMS Word Pairs Delayed Recall & .388 & .190 & .191 & -.061 & -.188 & -.282 & .076 & -.171 \\
\hline CMS Word Pairs Delayed Recognition & $.442^{t}$ & .178 & .148 & .098 & -.303 & .133 & .031 & .040 \\
\hline CMS Word Pairs Learning & .307 & $.515 *$ & .291 & .004 & -.349 & -.091 & -.442 & .096 \\
\hline CVLT-C Attention Span (Trial1) & $.441^{\mathrm{t}}$ & .227 & -.012 & -.087 & .254 & -.053 & .145 & -.405 \\
\hline CVLT-C Learning Efficiency (Trial5) & $.555 *$ & .049 & .014 & -.023 & .330 & $-.539^{t}$ & .242 & $-.450^{t}$ \\
\hline CVLT-C Total Learning (Total Trials) & $.503 *$ & .124 & .019 & -.017 & .443 & -.084 & .226 & -.170 \\
\hline CVLT-C Short-Delay Free Recall & $.674 * *$ & .208 & -.114 & .076 & $.525^{t}$ & -.135 & $.505^{t}$ & -.175 \\
\hline CVLT-C Short-Delay Cued Recall & $.660 * *$ & .300 & -.087 & .234 & .350 & -.294 & .287 & -.352 \\
\hline CVLT-C Long-Delay Free Recall & $.653 * *$ & .121 & -.079 & .059 & $.504^{t}$ & -.292 & $.515^{t}$ & $-.536^{\mathrm{t}}$ \\
\hline CVLT-C Long-Delay Cued Recall & $.580 *$ & .259 & -.152 & .142 & $.603 *$ & -.174 & $.512^{\mathrm{t}}$ & -.249 \\
\hline CVLT-C Delayed Recognition & .339 & -.070 & .035 & .225 & .171 & -.077 & .242 & .316 \\
\hline ROCF Delayed Spatial Recall & $.594 * *$ & .202 & -.110 & $.652 * *$ & .351 & .356 & $.508^{t}$ & .066 \\
\hline EMQ Spatial Memory \pm & $.495 *$ & $.500 *$ & -.099 & .120 & -.072 & -.010 & .191 & -.097 \\
\hline
\end{tabular}

( \pm : given that higher scores on the EMQ indicate poorer memory ability, the inverse correlations are reported so that they are comparable to other memory tests; $* * *=p<.001, * *=p<.01, *=p<.05,{ }^{\mathrm{t}}=p<.08$; two-tailed; CMS = Children's Memory Scale, CVLT-C = California Verbal Learning Test-Children's Version, ROCF = Rey-Osterrieth Complex Figure task, EMQ = Everyday Memory Questionnaire; Hippocampal volumes are presented as proportions of total intracranial volume) 
For the FASD group, left and right hippocampal volumes were associated with CVLT-C short- and long-delayed verbal recall, and right hippocampal volumes were associated with Rey-O delayed spatial recall. In controls, left hippocampal volumes correlated negatively with CVLT-C learning efficiency and right hippocampal volumes correlated negatively with CVLT-C learning efficiency and long-delay cued recall. However, further investigation revealed that these negative correlations may have been artificially inflated by one control participant who had the largest proportional hippocampal volumes and the significantly restricted range of the controls' CVLT-C learning efficiency scores, which may have led to spurious results.

\section{DISCUSSION}

This study examined verbal learning and verbal and spatial recall in relation to hippocampal volume in a sample of children with FASDs relative to controls. Results indicate that PAE was associated with significant impairments in intellectual ability, everyday spatial recall, and delayed reproduction of a spatial figure (ROCF). FASD participants also showed impaired verbal learning and recall using the CMS and CVLT-C, as evident by their poorer performance in immediate and delayed recall, delayed recognition, and verbal learning tasks relative to controls. In addition, the delayed verbal recall deficits observed in the FASD group appeared to be much greater on the CMS than on the CVLT-C tasks. This finding may be because of the larger number of learning trials on the CVLT-C than the CMS (i.e., 5 vs. 3), providing participants with more opportunities to encode and learn information, thus possibly leading to improved subsequent recall.

Although previous studies have found that verbal learning ability mediates the deficits in verbal recall associated with PAE, our results were mixed depending on the particular test administered. In the FASD group, deficits in delayed recall on the CVLT-C were no longer significant after controlling for learning efficiency, similar to the findings of Mattson and Roebuck (2002). However, unlike Willford et al. (2004), deficits in immediate and delayed recall on CMS Word Pairs in the FASD group remained significant (albeit reduced) after correcting for the initial amount of information learned. One possible explanation for this discrepancy is that Willford et al. (2004) examined CMS performance in a large sample of children with light to moderate PAE, whereas our sample included children referred for severe behavioral and cognitive problems who may have represented the extreme end of the ARND spectrum having greater deficits in verbal recall. Given that deficits in CVLT-C and CMS delayed recall were reduced after controlling for learning ability, our findings support the notion that in children with PAE, deficits in verbal learning contribute to impaired verbal recall.

A significant volume reduction in the left, but not right, hippocampus was found in FASD participants after controlling for total brain volume. This finding corresponds with the observations of Autti-Rämö et al. (2002); however, it is inconsistent with other studies reporting either reductions in both left and right hippocampal volumes (Riikonen et al., 2005) or relative sparing of hippocampal volume (Archibald et al., 2001) in children with FASDs. Because relatively few studies have attempted to examine hippocampal structure after PAE and because these studies provide conflicting results, our data should be interpreted with caution, because it remains unclear whether a specific pattern of hippocampal abnormality exists in children with FASDs.

Our finding of a group difference in intracranial volume is consistent with the extant literature showing that reduced brain size is commonly found among individuals with PAE. Similar to our results in controls, Lenroot and Giedd (2006) and Sowell et al. (2002b) reported that in typically developing children, total brain volume tends to be larger in boys than girls, and we also found this to be the case in children with FASDs. Additionally, we found significant associations between age and left and right hippocampal volumes in controls but not in FASD participants. Numerous studies of typically developing children have found increases in hippocampal volume throughout late childhood and adolescence, possibly because of increased myelination in the medial temporal region (Benes et al., 1994; Giedd et al., 1996; Suzuki et al., 2005). Our results suggest that in children with FASDs, hippocampal development continues to be adversely affected long after the initial PAE. However, because of our cross-sectional sample and limited sample size, these findings must be viewed as preliminary and warrant further investigation, especially using longitudinal designs.

Finally, we found positive structure-function correlations in children with FASDs, but not in controls. After controlling for age and IQ, we observed that larger left and right hippocampal volumes in FASD participants were associated with better short- and long-delayed verbal recall on the CVLT-C. In addition, delayed spatial recall (and to a small extent everyday spatial recall) in the FASD group appeared to be particularly associated with right hippocampal volumes, consistent with findings from other studies reporting associations between spatial recall deficits and right hippocampal damage (Bohbot et al., 1998; Uecker \& Nadel, 1996). It is important to note that most of the reported structure-function correlations in the FASD group are at a trend-level of significance, and due to the effects of multiple comparisons of memory function, these results should be interpreted with caution and viewed as exploratory, because further research is required in order to confirm these findings. We suspect that the lack of highly significant correlations in the present study may be due to the fact that structure-function relations may be more difficult to detect using structural MRI when compared to functional MRI, because volumetric measures provide no insight into the organizational processes of the hippocampus and associated neural networks involved in learning and memory (Van Petten, 2004). 
Similarly, hippocampal volume seems to have little relation to learning and recall performance in our control group. As Van Petten (2004) notes, hippocampal size and memory abilities vary significantly across healthy individuals, which may make it difficult to detect structure-function relationships especially when sample sizes are small. Perhaps structure-function relations are easier to detect in clinical populations with significant structural or functional damage to the hippocampus than in typically developing children whose hippocampal volumes fall within a "normal" range. Furthermore, other aspects of hippocampal integrity, such as shape and contour, as well as other structures within the medial temporal lobe (e.g., the entorhinal, parahippocampal, and perirhinal gyri) were not analyzed in the present study and may have a stronger relation to certain aspects of learning and memory than a singular measure of hippocampal volume in typically developing children.

This study contributes to the growing neuroimaging literature aimed at characterizing brain-behavior relationships in FASDs. However, a few limitations should be noted. The first is the relatively small sample sizes of each group, which is often common in neuroimaging studies but may have precluded a greater number of significant structurefunction relations and prevented us from analyzing our data by FASD subtype in order to determine whether more severe FASD forms contributed to either suboptimal memory performance or smaller hippocampal volumes. Another limitation is that our control participants may represent a nonrepresentative sample because of their higher-than-normal IQ levels and this may have biased their learning and recall performance or even their hippocampal and intracranial volumes relative to the FASD group. Finally, as is typical in studies of FASDs, our FASD group differed from controls on several environmental factors that may have adversely affected their cognitive and socioemotional development in ways we were unable to detect in the present study. For instance, the effects of PAE on hippocampal development and the acquisition of specific cognitive skills may have been further exacerbated by factors such as exposure to cigarettes or cocaine during pregnancy, early physical abuse or neglect, living in foster care, a diagnosis of ADHD, lower family income, lower birth weight, maternal stress, and poor prenatal care (Iqbal et al., 2005; May \& Gossage, 2001; Zhang et al., 2005).

\section{CONCLUSION}

In conclusion, we found that children with FASDs show significant impairments in verbal learning and verbal and spatial recall, reduced left hippocampal volumes, and a distinct pattern of association between hippocampal volume and verbal and spatial recall relative to typically developing control children. Future investigations using functional MRI are underway to examine whether hippocampal function during tests of verbal and spatial memory is specifically impaired in children with FASDs relative to controls. Overall, the present results confirm earlier findings of impaired verbal learning and spatial recall in children with FASDs and provide critical new information about hippocampal structure and its relation to learning and recall deficits in this clinical population.

\section{ACKNOWLEDGMENTS}

This research was originally conducted as one part of a larger New Emerging Team study (NET grant from the Canadian Institutes of Health Research \#54014; PI: James Brien, Queens University). The authors thank Meaghan Williamson for her work in recruitment and testing; Jovanka Skocic and Santosh Sekharan for their help with volumetric tracings; Dr. Susan Blaser who examined the neuroradiological scans; and Dianna Lanteigne and Victoria Martin for database management. The authors also thank the NET group for original planning and design, particularly Dr. Jim Brien and Dr. Gideon Koren, as well as the Motherisk Clinic team of Dr. Irena Nulman, Dr. Ellen Fantus, and Donna Sorbara, without whom this study would not have been possible. The authors are most grateful to the many parents/caregivers and their children for their participation in this study. Finally, the authors are indebted to three anonymous reviewers for their insightful and constructive comments. This work was additionally supported by a Canadian Institutes of Health Research operating grant (\#49488) to author JR, an Ontario Mental Health Foundation postdoctoral fellowship to author ES, a Children's Hospital of Eastern Ontario Foundation graduate scholarship to author KN, and an Ontario Graduate Scholarship and a Hospital for Sick Children Restracomp scholarship to author KW.

\section{REFERENCES}

Abel, E.L. \& Sokol, R.J. (1991). A revised conservative estimate of the incidence of FAS and its economic impact. Alcoholism: Clinical and Experimental Research, 15, 514-524.

Archibald, S.L., Fennema-Notestine, C., Gamst, A., Riley, E.P., Mattson, S.N., \& Jernigan, T.L. (2001). Brain dysmorphology in individuals with severe prenatal alcohol exposure. Developmental Medicine \& Child Neurology, 43, 148-154.

Autti-Rämö, I., Autti, T., Korkman, M., Kettunen, S., Salonen, O., \& Valanne, L. (2002). MRI findings in children with school problems who had been exposed prenatally to alcohol. Developmental Medicine and Child Neurology, 44, 98-106.

Benes, F.M., Turtle, M., Khan, Y., \& Farol, P. (1994). Myelination of a key relay zone in the hippocampal formation occurs in the human brain during childhood, adolescence, and adulthood. Archives of General Psychiatry, 51, 477-484.

Berman, R.F. \& Hannigan, J.H. (2000). Effects of prenatal alcohol exposure on the hippocampus: Spatial behavior, electrophysiology and neuroanatomy. Hippocampus, 10, 94-110.

Bohbot, V.D., Kalina, M., Stepankova, K., Spackova, N., Petrides, M., \& Nadel, L. (1998). Spatial memory deficits in patients with lesions to the right hippocampus and to the right parahippocampal cortex. Neuropsychologia, 36, 1217-1238.

Chudley, A.E., Conry, J., Cook, J.L., Loock, C., Rosales, T., \& LeBlanc, N. (2005). Fetal alcohol spectrum disorder: Canadian guidelines for diagnosis. Canadian Medical Association Journal, 172, S1-S21.

Clarren, S.K. \& Smith, D.W. (1978). The fetal alcohol syndrome. New England Journal of Medicine, 298, 1063-1067. 
Cohen, M.J. (1997). Children's Memory Scale Manual. San Antonio, TX: The Psychological Corporation.

Cornish, I.M. (2000). Factor structure of the Everyday Memory Questionnaire. British Journal of Psychology, 91, 427-438.

Delis, D.C., Kramer, J.H., Kaplan, E., \& Ober, B.A. (1994). California Verbal Learning Test-Children's Version. San Antonio, TX: The Psychological Corporation.

Donders, J. (1999). Performance discrepancies on the California Verbal Learning Test-Children's Version in the standardization sample. Journal of the International Neuropsychological Society, 5, 26-31.

Ghaem, O., Mellet, E., Crivello, F., Tzourio, N., Mazoyer, B., Berthoz, A., \& Denis, M. (1997). Mental navigation along memorized routes activates the hippocampus, precuneus, and insula. Neuroreport, 8, 739-744.

Gianoulakis, C. (1990). Rats exposed prenatally to alcohol exhibit impairment in spatial navigation test. Behavioural Brain Research, 36, 217-228.

Giedd, J.N., Vaituzis, A.C., Hamburger, S.D., Lange, N., Rajapakse, J.C., Kaysen, D., Vauss, Y.C., \& Rapoport, J.L. (1996). Quantitative MRI of the temporal lobe, amygdala, and hippocampus in normal human development: Ages 4-18 years. The Journal of Comparative Neurology, 366, 223-230.

Goodlett, C.R. \& Johnson, T.B. (1999). Temporal windows of vulnerability within the third trimester equivalent: Why "knowing when" matters. In J.H. Hannigan, C.R. Goodlett, L.P. Spear, \& N.E. Spear (Eds.), Alcohol and alcoholism: Effects on brain and development (pp. 59-91). Mahwah, NJ: L. Earlbaum Associates.

Greenbaum, R., Nulman, I., Rovet, J., \& Koren, G. (2002). The Toronto experience in diagnosing alcohol-related neurodevelopmental disorder: A unique profile of deficits and assets. Canadian Journal of Pharmacology, 9, 215-225.

Griffiths, S.Y., Sherman, E.M.S., Slick, D.J., Lautzenhiser, A., Westerveld, M., \& Zaroff, C.M. (2006). The factor structure of the CVLT-C in pediatric epilepsy. Child Neuropsychology, 12, 191-203.

Hardwood, H. (2000). Updating estimates of the economic costs of alcohol abuse in the United States: Estimates, update methods, and data. Report prepared by The Lewin Group for the National Institute on Alcohol Abuse and Alcoholism.

Iqbal, U., Brien, J.F., Banjanin, S., Andrews, M.H., Matthews, S.G., \& Reynolds, J.N. (2005). Chronic prenatal ethanol exposure alters glucocorticoid signalling in the hippocampus of the postnatal guinea pig. Journal of Neuroendocrinology, 17, 600-608

Isaacs, E.B., Lucas, A., Chong, W.K., Wood, S.J., Johnson, C.L., Marshall, C., Vargha-Khadem, F., \& Gadian, D.G. (2000). Hippocampal volume and everyday memory in children of very low birth weight. Pediatric Research, 47, 713-720.

Jones, K.L. \& Smith, D.W. (1973). Recognition of the fetal alcohol syndrome in early infancy. Lancet, 2, 999-1001.

Klintsova, A.Y., Helfer, J.L, Calizo, L.H., Dong, W.K., Goodlett, C.R., \& Greenough, W.T. (2007). Persistent impairment of hippocampal neurogenesis in young adult rats following early postnatal alcohol exposure. Alcoholism: Clinical and Experimental Research, 31, 2073-2082.

Kodituwakku, P.W. (2007). Defining the behavioral phenotype in children with fetal alcohol spectrum disorders: A review. Neuroscience and Biobehavioral Reviews, 31, 192-201.

Lenroot, R.K. \& Giedd, J.N. (2006). Brain development in children and adolescents: Insights from anatomical magnetic res- onance imaging. Neuroscience and Biobehavioral Reviews, 30 , $718-729$.

Livy, D., Miller, E.K., Maier, S.E., \& West, J.R. (2003). Fetal alcohol exposure and temporal vulnerability: Effects of bingelike alcohol exposure on the developing rat hippocampus. Neurotoxicology \& Teratology, 25, 447-458.

Maguire, E.A., Burgess, N., Donnett, J.G., Frackowiak, R.S.J, Frith, C.D., \& O'Keefe, J. (1998). Knowing where and getting there: A human navigation network. Science, 280, 921-924.

Malisza, K.L., Allman, A., Shiloff, D., Jakobson, L., Longstaffe, S., \& Chudley, A.E. (2005). Evaluation of spatial working memory function in children and adults with fetal alcohol spectrum disorders: A functional magnetic resonance imaging study. Pediatric Research, 58, 1150-1157.

Mattson, S.N. \& Riley, E.P. (1998). A review of the neurobehavioral deficits in children with fetal alcohol syndrome or prenatal exposure to alcohol. Alcoholism: Clinical and Experimental Research, 22, 279-294.

Mattson, S.N., Riley, E.P., Delis, D.C., Stern, C., \& Jones, K.L. (1996). Verbal learning and memory in children with fetal alcohol syndrome. Alcoholism: Clinical and Experimental Research, 20, 810-816.

Mattson, S.N., Riley, E.P., Gramling, L., Delis, D.C., \& Jones, K.L. (1998). Neuropsychological comparison of alcoholexposed children with or without physical features of fetal alcohol syndrome. Neuropsychology, 12, 146-153.

Mattson, S.N. \& Roebuck, T.M. (2002). Acquisition and retention of verbal and nonverbal information in children with heavy prenatal alcohol exposure. Alcoholism: Clinical and Experimental Research, 26, 875-882

May, P.A., Fiorentino, D., Gossage, J.P., Kalberg, W.O., Hoyme, H.E., Robinson, L.K., Coriale, G., Jones, K.L., del Campo, M., Tarani, L., Romeo, M., Kodituwakku, P.W., Deiana, K., Buckley, D., \& Ceccanti, M. (2006). Epidemiology of FASD in a province in Italy: Prevalence and characteristics of children in a random sample of schools. Alcoholism: Clinical and Experimental Research, 30, 1562-1575.

May, P.A. \& Gossage, J.P. (2001). Estimating the prevalence of fetal alcohol syndrome: A summary. Alcohol Research \& Health, $25,159-167$.

Niccols, A. (2007). Fetal alcohol syndrome and the developing socio-emotional brain. Brain and Cognition, 65, 135-142.

O'Hare, E.D., Kan, E., Yoshii, J., Mattson, S.N., Riley, E.P., Thompson, P.M., Toga, A.W., \& Sowell, E.R. (2005). Mapping cerebellar vermal morphology and cognitive correlates in prenatal alcohol exposure. Neuroreport, 16, 1285-1290.

Osterrieth, P. \& Rey, A. (1944). Le test de copie d'une figure complex. Archives de Psychologie, 30, 205-221.

Rey, A. (1942). L'examen psychologique dans les cas d'encephalopathie traumatique. Archives de Psychologie, 28, 286-340.

Riikonen, R.S., Nokelainen, P., Valkonen, K., Kolehmainen, A.I., Kumpulainen, K.I., Könönen, M., Vanninen, R.L., \& Kuikka, J.T. (2005). Deep serotonergic and dopaminergic structures in fetal alcoholic syndrome: A study with nor- $\beta$-CIT-singlephoton emission computed tomography and magnetic resonance imaging volumetry. Biological Psychiatry, 57, 1565-1572.

Riikonen, R.S., Salonen, I., Partanen, K., \& Verho, S. (1999). Brain perfusion SPECT and MRI in fotal alcohol syndrome. Developmental Medicine \& Child Neurology, 41, 652-659.

Riley, E.P. \& McGee, C.L. (2005). Fetal alcohol spectrum disorders: An overview with emphasis on changes in brain and behavior. Experimental Biology and Medicine, 230, 357-365. 
Robinson, G.C., Conry, J.L., \& Conry, R.F. (1987). Clinical profile and prevalence of fetal alcohol syndrome in an isolated community in British Columbia. Canadian Medical Association Journal, 137, 203-207.

Salorio, C.F., Slomine, B.S., Grados, M.A., Vasa, R.A., Christensen, J.R., \& Gerring, J.P. (2005). Neuroanatomic correlates of CVLT-C performance following pediatric traumatic brain injury. Journal of the International Neuropsychological Society, 11, 686-696.

Sampson, P.D., Streissguth, A.P., Bookstein, F.L., Little, R.E., Clarren, S.K., Dehaene, P., Hanson, J.W., \& Graham, J.M. (1997). Incidence of fetal alcohol syndrome and prevalence of alcoholrelated neurodevelopmental disorder. Teratology, 56, 317-326.

Sowell, E.R., Lu, L.H., O'Hare, E.D., McCourt, S.T., Mattson, S.N., O'Connor, M.J., \& Bookheimer, S.Y. (2007). Functional magnetic resonance imaging of verbal learning in children with heavy prenatal alcohol exposure. Neuroreport, 18, 635-639.

Sowell, E.R., Mattson, S.N., Kan, E., Thompson, P.M., Riley, E.P., \& Toga, A.W. (2008). Abnormal cortical thickness and brainbehavior correlation patterns in individuals with heavy prenatal alcohol exposure. Cerebral Cortex, 18, 136-144.

Sowell, E.R., Mattson, S.N., Thompson, P.M., Jernigan, T.L., Riley, E.P., \& Toga, A.W. (2001). Mapping callosal morphology and cognitive correlates: Effects of heavy prenatal alcohol exposure. Neurology, 57, 235-244.

Sowell, E.R., Thompson, P.M., Mattson, S.N., Tessner, K.D., Jernigan, T.L., Riley, E.P., \& Toga, A.W. (2002a). Regional brain shape abnormalities persist into adolescence after heavy prenatal alcohol exposure. Cerebral Cortex, 12, 856-865.

Sowell, E.R., Trauner, D.A., Gamst, A., \& Jernigan, T.L. (2002b). Development of cortical and subcortical brain structures in childhood and adolescence: A structural MRI study. Developmental Medicine \& Child Neurology, 44, 4-16.

Spreen, O. \& Strauss, E. (1998). A Compendium of Neuropsychological Tests: Administration, Norms, and Commentary. $\left(2^{\text {nd }}\right.$ ed.). New York: Oxford University Press.

Square, D. (1997). Fetal alcohol syndrome epidemic on Manitoba reserve. Canadian Medical Association Journal, 157, 59-60.

Stade, B., Ungar, W.J., Stevens, B., Beyene, J., \& Koren, G. (2007). Cost of fetal alcohol spectrum disorder in Canada. Canadian Family Physician, 53, 1303-1304.

Stratton, K., Howe, C., \& Battaglia, F. (1996). Fetal Alcohol Syndrome: Diagnosis, Epidemiology, Prevention, and Treatment. Washington, DC: National Academy Press.

Streissguth, A.P., Aase, J.M., Clarren, S.K., Randels, S.P., LaDue, R.A., \& Smith, D.F. (1991). Fetal Alcohol Syndrome in ado- lescents and adults. The Journal of the American Medical Association, 265, 1961-1967.

Sunderland, A., Harris, J.E., \& Baddeley, A.D. (1983). Assessing everyday memory after severe head injury. In J.E. Harris \& P.E. Morris (Eds.), Everyday memory, actions, and absentmindedness (pp. 191-206). London: Academic Press.

Sutherland, R.J., McDonald, R.J., \& Savage, D.D. (2000). Prenatal exposure to moderate levels of ethanol can have long-lasting effects on learning and memory in adult offspring. Psychobiology, 28, 532-539.

Suzuki, M., Hagino, H., Nohara, S., Zhou, S., Kawasaki, Y., Takahashi, T., Matsui, M., Seto, H., Ono, T., \& Kurachi, M. (2005). Male-specific volume expansion of the human hippocampus during adolescence. Cerebral Cortex, 15, 187-193.

Tomlinson, D., Wilce, P., \& Bedi, K.S. (1998). Spatial learning ability of rats following differing levels of exposure to alcohol during early postnatal life. Physiology \& Behavior, 63, 205-211.

Uecker, A. \& Nadel, L. (1996). Spatial locations gone awry: Object and spatial memory deficits in children with fetal alcohol syndrome. Neuropsychologia, 34, 209-223.

Van Petten, C. (2004). Relationship between hippocampal volume and memory ability in healthy individuals across the lifespan: Review and meta-analysis. Neuropsychologia, 42, 1394-1413.

Vargha-Khadem, F., Gadian, D.G., Watkins, K.E., Connelly, A., Van Paesschen, W., \& Mishkin, M. (1997). Differential effects of early hippocampal pathology on episodic and semantic memory. Science, 277, 376-380.

Viljoen, D.L., Gossage, J.P., Brooke, L., Adnams, C.M., Jones, K.L., Robinson, L.K., Hoyme, H.E., Snell, C., Khaole, N.C., Kodituwakku, P., Asante, K.O., Findlay, R., Quinton, B., Marais, A.S., Kalberg, W.O., \& May, P.A. (2005). Fetal alcohol syndrome epidemiology in a South African community: A second study of a very high prevalence area. Journal of Studies on Alcohol, 66, 593-604.

Wechsler, D. (1999). Wechsler Abbreviated Scale of Intelligence. New York: Psychological Corporation.

Willford, J.A., Richardson, G.A., Leech, S.L., \& Day, N.L. (2004). Verbal and visuospatial learning and memory function in children with moderate prenatal alcohol exposure. Alcoholism: Clinical and Experimental Research, 28, 497-507.

Zhang, X., Sliwowska, J.H., \& Weinberg, J. (2005). Prenatal alcohol exposure and fetal programming: Effects on neuroendocrine and immune function. Experimental Biology and Medicine, 230, 376-388. 